\title{
Erratum to: A prospective feasibility study of primary prophylaxis against invasive fungal disease with voriconazole following umbilical cord blood transplantation with fludarabine- based conditioning
}

\author{
Shinsuke Takagi $\cdot$ Hideki Araoka $\cdot$ Naoyuki Uchida $\cdot$ Yumiko Uchida Daisuke Kaji Hikari Ota \\ Aya Nishida $\cdot$ Kazuya Ishiwata $\cdot$ Masanori Tsuji $\cdot$ Hisashi Yamamoto $\cdot$ Tadaaki Ito $\cdot$ Naofumi Matsuno \\ Go Yamamoto · Yuki Asano-Mori • Masahiro Hayashi · Koji Izutsu $\cdot$ Kazuhiro Masuoka $\cdot$ Atsushi Wake • \\ Shigeyoshi Makino $\cdot$ Akiko Yoneyama $\cdot$ Shuichi Taniguchi
}

Published online: 17 April 2014

(C) The Japanese Society of Hematology 2014

\section{Erratum to: Int J Hematol}

DOI 10.1007/s12185-014-1529-7

The authors would like to correct the error in the publication of the original article. The corrected detail is given below for your reading.

\begin{abstract}
Acknowledgments The authors thank all physicians, nurses, pharmacists, transplantation coordinator (Ms. Madoka Narita), data managers (Ms. Naomi Yamada, Ms. Rumiko Tsuchihashi and Ms. Kaoru Kobayashi), and support personnel for their compassionate care of the patients involved in this study, which was supported in part by the Japanese Ministry of Health, Labor and Welfare (Research Grant for Allergic Disease and Immunology H20-015).
\end{abstract}

The online version of the original article can be found under doi:10.1007/s12185-014-1529-7.

S. Takagi $(\bowtie) \cdot$ N. Uchida - D. Kaji · H. Ota · K. Ishiwata .

M. Tsuji · H. Yamamoto · G. Yamamoto · Y. Asano-Mori ·

$\mathrm{K}$. Izutsu $\cdot \mathrm{S}$. Taniguchi

Department of Hematology, Toranomon Hospital,

2-2-2 Toranomon, Minato-ku, Tokyo 105-0001, Japan

e-mail: shinsuke-takagi@umin.net

S. Takagi $\cdot$ A. Nishida $\cdot$ N. Matsuno $\cdot$ A. Wake

Department of Hematology, Toranomon Hospital Kajigaya,

Kanagawa, Japan

H. Araoka - A. Yoneyama

Department of Infectious Diseases, Toranomon Hospital,

Tokyo, Japan

Y. Uchida $\cdot$ T. Ito $\cdot$ M. Hayashi

Department of Pharmacy, Toranomon Hospital, Tokyo, Japan

H. Ota $\cdot$ S. Makino

Department of Transfusion Medicine, Toranomon Hospital,

Tokyo, Japan

K. Masuoka

Department of Hematology, Mishuku Hospital, Tokyo, Japan

S. Taniguchi

Okinaka Memorial Institute for Medical Research, Tokyo, Japan 